\title{
COMISIÓN INTERGESTORA REGIONAL: ¿ESPACIO LOCAL DE CONCERTACIÓN Y ARTICULACIÓN INTER-FEDERATIVA?
}

\section{ARTÍCULO ORIGINAL}

SANTOS, Nadja Romeiro dos ${ }^{1}$, COTA, Ana Lídia Soares ${ }^{2}$

SANTOS, Nadja Romeiro dos. COTA, Ana Lídia Soares. Comisión intergestora regional: ¿espacio local de concertación y articulación inter-federativa? Revista Científica Multidisciplinar Núcleo do Conhecimento. Año 06, Ed. 09, Vol. 03, págs. 173-189. Septiembre 2021. ISSN: 2448-0959, Enlace de acceso: https://www.nucleodoconhecimento.com.br/tecnologia-es/comision-intergestora, DOI: 10.32749/nucleodoconhecimento.com.br/tecnologia-es/comision-intergestora

\section{RESUMEN}

La Comisión Regional de Intergerentes (CIR) configura el espacio de acuerdo, articulación, cooperación y fortalecimiento de la región de la salud. El objetivo de este estudio fue reflexionar sobre las disposiciones normativas que subyacen a la construcción de la comisión como lugar de consolidación de la gestión regional atenuante de los conflictos interfederantes. Teniendo como guía la pregunta: ¿cómo proporcionan las Comisiones Regionales de Gestión espacios locales de acuerdo y articulación interfederativa? Estudio cualitativo, basado en investigación documental, basado en el análisis de las resoluciones de las Comisiones Regionales Intergestión de Alagoas, correspondientes al periodo 2017 a 2019, tondo como estándar de referencia el Decreto 7.508/11. Los resultados se organizaron en cuatro categorías: Planificación Regional de Salud; organización de acciones y servicios de atención de la salud: región de la salud; Federalismo, CIR y financiamiento de los servicios de

\footnotetext{
${ }^{1}$ Estudiante de doctorado en el Programa de Postgrado en Sociedad, Tecnologías y Políticas Públicas. Centro Universitario Tiradentes (UNIDAD).

${ }^{2}$ Doctora en Odontopediatría, Profesora del Curso de Odontología y del Programa de Posgrado en Sociedad, Tecnologías y Políticas Públicas (UNIT / AL) Centro Universitario Tiradentes.
}

RC: 98470

Disponible: https://www.nucleodoconhecimento.com.br/tecnologia-es/comisionintergestora 
salud; gobernanza y CIR: canal de negociación y decisión entre los gestores municipales y el Estado dentro de la Región Sanitaria. Las Comisiones Regionales De Gestión desarrollan un trabajo significativo en las discusiones de políticas regionales, siendo una herramienta fundamental para el fortalecimiento de la gobernanza en los territorios, mediante la negociación de la asignación y distribución de recursos, esenciales para la planificación, los pactos interfederativos y el financiamiento de la salud, desempeñando un papel clave para el sistema regional de salud de Alagoas, espacio democrático, político y cooperativo.

Palabras clave: Regionalización, Gestión de la Salud, Sistema Único de Salud, Federalismo, Política de Salud.

\section{INTRODUCCIÓN}

Las Políticas de Salud del Brasil han sido formuladas para dar cumplimiento al artículo 196 de la Constitución Federal (CF/1988), sobre la base de acciones y servicios de salud pública que forman parte de una red regionalizada y jerárquica, constituyendo un sistema único (SUS), organizado de acuerdo con diversos lineamientos, incluyendo la descentralización, la atención integral y la participación comunitaria (Artículo 198) (BRASIL, 1988).

Un avance importante en este sentido fue la regulación de la Ley Orgánica de Salud (Ley 8.080/90) a través del Decreto Federal № 7.508 publicado en julio de 2011 consideró el marco regulatorio densificando las disposiciones constitucionales y legales relacionadas con la realización del derecho fundamental a la salud en Brasil, nodits sobre la descentralización de la salud pública, con la organización del SUS, la planificación de acciones de atención de salud y la articulación interfederante.

Uno de los principales logros se refiere a la operacionalización del SUS dentro del territorio regional, desde la validación de los espacios de acuerdo y deliberación, con miras a la definición de reglas de gestión compartida, denominadas Comisiones

RC: 98470

Disponible: https://www.nucleodoconhecimento.com.br/tecnologia-es/comisionintergestora 
Regionales De Gestión (CIR), siendo el proceso de descentralización de la salud pública, una de las premisas constitucionales, se intensifica con la legitimación de espacios regionales de acuerdo.

Este proceso político-administrativo del SUS en Brasil, modelo victorioso en la Asamblea Nacional Constituyente (1986-1988), fue el consectario de una de las principales demandas del Movimiento de Reforma Sanitaria, construida debido a las diversas críticas al modelo centralizador entonces vigente en el país, ejecutada en su momento por el Instituto Nacional de Asistencia Médica de la Seguridad Social (INAMPS).

Este principio organizativo también se incorporó a las demás políticas públicas instituidas, especialmente la salud, la educación y la asistencia social, que, en mutua articulación, estructuran las bases de un Estado de protección social.

Según Wagner (2006), la descentralización tiende a producir una autonomía de decisión en relación con la salud para las entidades federadas. La creciente centralización financiera del Estado, junto con el ejercicio continuo de la regulación fiscal de los estados y municipios, y un papel decisivo en la formulación de políticas produjo una combinación de centralización estatal, con políticas sectoriales descentralizadoras que, en el caso de la salud, siempre dependieron de la inducción federal (VIANA et al, 2002).

La región se entiende como el espacio geográfico continuo, formado por conglomerados de municipios vecinos, definidos a partir de la existencia de identidades culturales, sociales y económicas y otras, con el objetivo de integrar, organizar, planificar y ejecutar acciones y servicios de salud (BRASIL, 2011).

Nesse ambiente, o CIR se destaca como instrumento de apoio à implementação de políticas de saúde nas regiões federativas. $\mathrm{Na}$ prática, funcionamos como instituições regionais de governança das redes de atenção à saúde, cuja sintonia é

RC: 98470

Disponible: https://www.nucleodoconhecimento.com.br/tecnologia-es/comisionintergestora 
relevante para realizar a dinâmica da regionalização, identificar problemas, definir prioridades e fornecer soluções para a sua organização, auxiliando na constituição de objetivos comuns e não no estabelecimento de uma unidade lógica passou a ser da população e a prestação de dois serviços de saúde.

Es en el contexto de mantener el protagonismo municipal, y fortalecer las relaciones de interdependencia y corresponsabilidad sanitaria entre las entidades estatales y municipales, que surge el CIR, teniendo como misión la gestión de la Política Regional de Salud, basada en el conocimiento de la realidad epidemiológica y socioeconómica de los municipios vecinos.

Con respecto al estado de Alagoas, el CIR coordina la construcción de la Red Regional de Atención (RAR), asegurando el acceso de la población a los servicios de salud regulando, monitoreando y evaluando las metas establecidas para los municipios insertos en su área.

Por ello, este artículo pretende reflexionar sobre las disposiciones normativas que subyacen a la construcción del CIR como espacios de consolidación de la gestión regional atenuando los conflictos interfederantes, analizando la regionalización en el SUS, buscando verificar la influencia de la cooperación intergubernamental en el fortalecimiento de esta estrategia. Para apoyar el análisis propuesto, se revisaron las referencias teóricas sobre el tema, a partir de la visión general de los fundamentos del federalismo, el SUS en la estructura federativa brasileña y los conceptos de descentralización y regionalización, terminando con la discusión de algunos factores críticos. En vista de las reflexiones anteriores, surgió la siguiente pregunta: ¿cómo proporciona el CIR espacios locales de acuerdo y articulación interfederativa?

\section{METODOLOGÍA}

Se trata de un estudio descriptivo y exploratorio con enfoque cualitativo, realizado a través de una revisión y análisis temático del contenido de los documentos oficiales

RC: 98470

Disponible: https://www.nucleodoconhecimento.com.br/tecnologia-es/comisionintergestora 
del CIR/AL, como espacio de concordancia y articulación interfederativa del SUS, permitiendo agregar conocimientos sobre el objeto estudiado, analizando también los avances de las políticas de salud pública desde el proceso de regionalización (MINAYO, 2010).

Analizamos las resoluciones elaboradas por el CIR, correspondientes al período 2017 a 2019, a disposición del público en la página del Diario Oficial del Estado de Alagoas (DOE/AL) http://www.imprensaoficial.com.br/diario-oficial, utilizando como estándar de referencia el Decreto 7.508/11. Los hallazgos se sistematizaron en cuatro categorías analíticas, agrupadas después de la lectura de los documentos, cuyos hallazgos están ordenados en la Tabla 1, es decir: planificación (Categoría 1); organización (Categoría 2); (Categoría 3) y gobernanza (Categoría 4).

\section{RESULTADOS}

Del total de 192 resoluciones leídas, se generó un marco analítico (Tabla 1), que ordenó las principales deliberaciones del CIR y su respectivo significado, como contenidos relevantes de las categorías previamente elegidas, para guiar la discusión.

Tabla I: Caracterización de resoluciones por categorías de análisis para el periodo 2017-2019.

\begin{tabular}{|c|c|c|}
\hline Categoría & $\begin{array}{l}\text { Sentido de } \\
\text { deliberación }\end{array}$ & Principales deliberaciones \\
\hline Planificación & $\begin{array}{lr}\text { Estrategias } & \text { de } \\
\text { cooperación } & \text { que } \\
\text { contribuyan } & \text { al } \\
\text { acuerdo, } & \end{array}$ & $\begin{array}{l}\text { Aprueba metas propuestas por las áreas } \\
\text { técnicas del Departamento de Estado de } \\
\text { Salud - SESAU referidas a indicadores } \\
\text { interfederativos para el periodo } 2017-2021\end{array}$ \\
\hline & $\begin{array}{l}\text { garantizando el } \\
\text { acceso a los }\end{array}$ & $\begin{array}{l}\text { Aprueba la planificación de la salud, la } \\
\text { atención médica y la articulación }\end{array}$ \\
\hline
\end{tabular}

RC: 98470

Disponible: https://www.nucleodoconhecimento.com.br/tecnologia-es/comisionintergestora 


\begin{tabular}{|c|c|c|}
\hline & \multirow{6}{*}{$\begin{array}{l}\text { servicios de salud a } \\
\text { la población. } \\
\text { Estrategia que } \\
\text { favorezca la calidad } \\
\text { a la implementación } \\
\text { de políticas de } \\
\text { salud. }\end{array}$} & interfederante \\
\hline & & Aprueba Plan Estatal de Humanización de la \\
\hline & & $\begin{array}{l}\text { Salud del Estado de Alagoas, cuatrio } 2016 \text { - } \\
2019 \text { y plan operativo } 2017\end{array}$ \\
\hline & & $\begin{array}{l}\text { Objetivos de Reactua de indicadores } \\
\text { interfederativos. }\end{array}$ \\
\hline & & $\begin{array}{l}\text { Modifica Plan Estatal de la Red de Atención } \\
\text { a la Discapacidad para la inserción de } \\
\text { incentivos económicos a la inversión del } \\
\text { Ministerio de Salud }\end{array}$ \\
\hline & & $\begin{array}{l}\text { Aprueba el plan de acción de servicio como } \\
\text { referencia para la macrorregión de estado }\end{array}$ \\
\hline \multirow[t]{8}{*}{ Organización } & \multirow{8}{*}{$\begin{array}{l}\text { Organización de } \\
\text { servicios que } \\
\text { favorezca el } \\
\text { fortalecimiento de la } \\
\text { red de salud en la } \\
\text { región. }\end{array}$} & Aprueba la habilitación del servicio \\
\hline & & $\begin{array}{l}\text { Aprueba mapa de vinculación de referencias } \\
\text { de laboratorio para la realización de pruebas } \\
\text { citopatológicas para el cribado de cáncer de } \\
\text { cuello uterino. }\end{array}$ \\
\hline & & $\begin{array}{l}\text { Aprueba el Manual de Tratamiento Fuera de } \\
\text { Casa - TFD Alagoas. }\end{array}$ \\
\hline & & $\begin{array}{l}\text { Aprueba la implementación de camas de } \\
\text { UCl dentro de la Red de Emergencia y } \\
\text { Emergencia (RUE) }\end{array}$ \\
\hline & & $\begin{array}{l}\text { Aprueba redistribución de camas de salud } \\
\text { mental en región salud }\end{array}$ \\
\hline & & $\begin{array}{l}\text { Aprueba la inserción de la Unidad de } \\
\text { Atención de Emergencia Porte III }\end{array}$ \\
\hline & & $\begin{array}{l}\text { Aprueba la implementación de la Unidad de } \\
\text { Cuidados de Largo Plazo - UCP }\end{array}$ \\
\hline & & Aprobada la calificación de camas traseras \\
\hline
\end{tabular}

RC: 98470

Disponible: https://www.nucleodoconhecimento.com.br/tecnologia-es/comisionintergestora 


\begin{tabular}{|c|c|c|}
\hline & & $\begin{array}{l}\text { de la Red de Emergencias y Emergencias } \\
\text { (RUE), }\end{array}$ \\
\hline & & Aprueba la recalificación del servicio \\
\hline & & $\begin{array}{l}\text { Aprueba la implementación de centro de } \\
\text { atención de urgencias para pacientes con } \\
\text { ictus, Tipo III, en un servicio de salud, como } \\
\text { referencia para la región sanitaria }\end{array}$ \\
\hline & & $\begin{array}{l}\text { Aprueba el cambio de la especialidad del } \\
\text { servicio Puerta de Entrada de Urgencia } \\
\text { Clínica. }\end{array}$ \\
\hline Financiación & $\begin{array}{l}\text { Estrategia de } \\
\text { financiación y } \\
\text { fomento de la } \\
\text { regionalización. } \\
\text { Implementación de } \\
\text { políticas públicas en }\end{array}$ & $\begin{array}{l}\text { Aprueba la reubicación de los límites } \\
\text { máximos físicos y financieros en la } \\
\text { programación acordada e integrada } \\
\text { Financiamiento y transferencia de recursos } \\
\text { federales - Red de atención de emergencias } \\
\text { y emergencias }\end{array}$ \\
\hline & la región. & $\begin{array}{l}\text { Aprueba la reubicación de recursos } \\
\text { financieros para la transferencia del SUS del } \\
\text { Bloque de Costeo de Acciones y Servicios } \\
\text { de Salud Pública del municipio, referido al } \\
\text { uso de techos físicos y financieros de } \\
\text { acuerdo a la programación acordada e } \\
\text { integrada de atención de mediana y alta } \\
\text { complejidad (Ambulatorio y Hospital MAC) }\end{array}$ \\
\hline & & $\begin{array}{l}\text { Aprueba la solicitud de incremento de los } \\
\text { recursos financieros de Mediana y Alta } \\
\text { Complejidad Ambulatoria y Hospitalaria } \\
\text { (MAC) del municipio. }\end{array}$ \\
\hline & & Estrategias y Compensación - FAEC para el \\
\hline
\end{tabular}

RC: 98470

Disponible: https://www.nucleodoconhecimento.com.br/tecnologia-es/comisionintergestora 


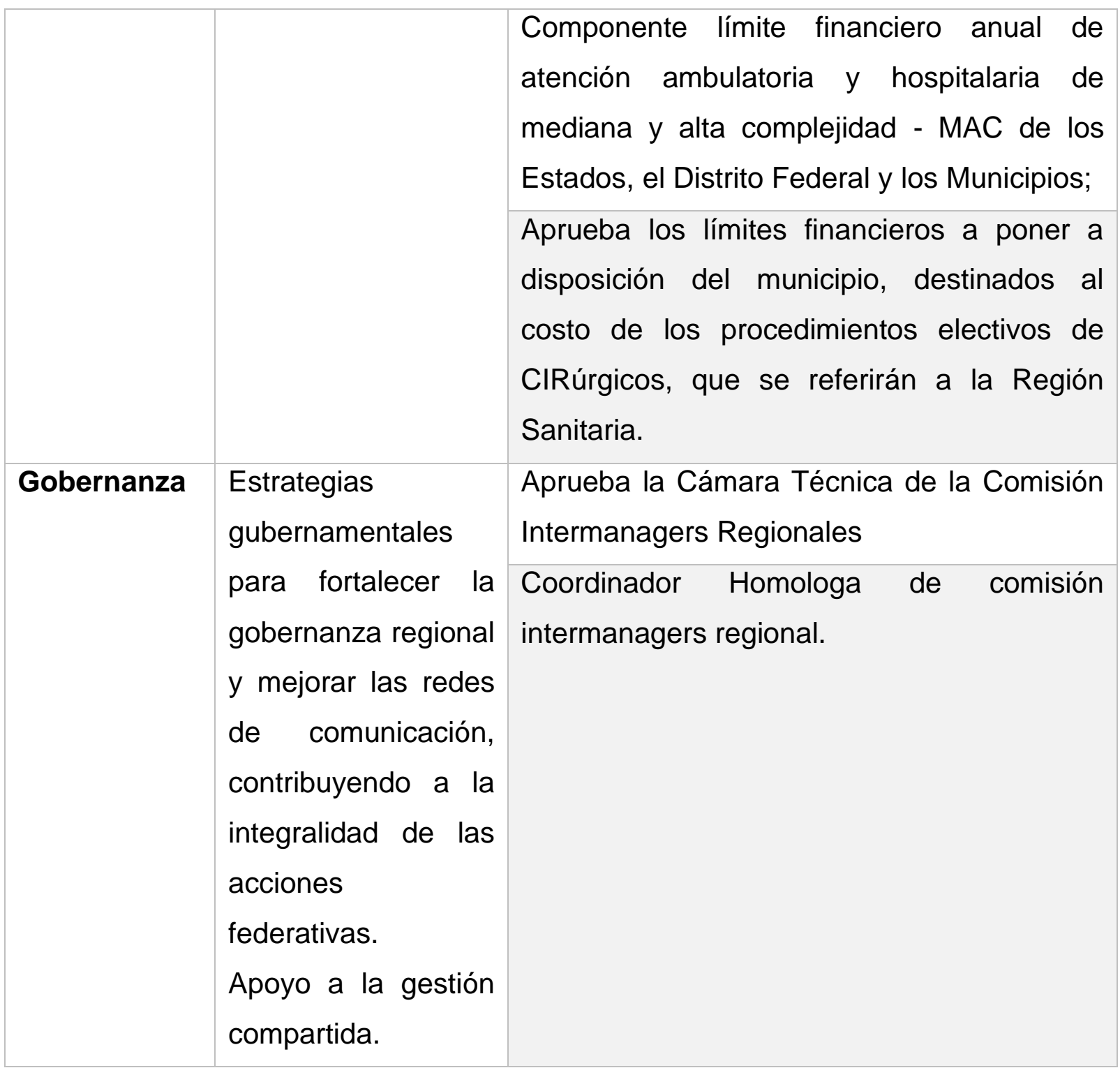

Fuente: Autoría propia, 2020

\section{DISCUSIÓN}

\subsection{PLANIFICACIÓN SANITARIA REGIONAL}

La planificación es un proceso permanente y dinámico, y también debe incluir situaciones de emergencia consideradas a corto plazo. En el área de la salud, la planificación es competencia de las tres esferas de Gobierno y constituye la base

RC: 98470

Disponible: https://www.nucleodoconhecimento.com.br/tecnologia-es/comisionintergestora 
para la ejecución, seguimiento, evaluación, control y gestión del sistema de salud, asumiendo el conocimiento de la realidad existente y de los instrumentos disponibles y necesarios para alcanzar las metas propuestas.

Según Santos (2017) la ausencia de planificación perjudica la organización de los servicios de salud, debilitando la cooperación y coordinación de la política de regionalización. De hecho, la Constitución Federal, en su art. 176, determina que es obligatoria la planificación para el poder público e inductor para el sector privado (BRASIL, 1988).

El proceso de regionalización de la salud permite la asignación de servicios en la red de salud, con el fin de contemplar todos los niveles de salud, de acuerdo a las necesidades de los individuos. Los documentos analizados en esta investigación demostraron que la planificación se ha convertido en una agenda importante en las agendas de discusión de los gestores en las regiones de salud.

Una de las consecuencias de la planificación es aclarar las responsabilidades de las tres esferas de gobierno, la definición de la prestación y gestión de servicios y la contribución de recursos financieros. De esta manera, el proceso de planificación y presupuestación debe ser indivisible. Por lo tanto, la programación presupuestaria en salud es esencial, como parte integral y detallada de los planes de salud (BRASIL, 2011).

Según Duarte y otros colaboradores (2018) la organización de la planificación y presupuesto del SUS es ascendente, integrada realizada de manera regionalizada, desde el reconocimiento de los problemas de salud de cada municipio, construida a través de la articulación entre entidades autónomas, a fin de asegurar la calidad de vida de la población, a través de la organización de los servicios de salud. En la práctica, la planificación y organización de las actividades de cada nivel de dirección del SUS será ascendente, guiada por las necesidades de salud de la población, a

RC: 98470

Disponible: https://www.nucleodoconhecimento.com.br/tecnologia-es/comisionintergestora 
través de la organización del plan de salud en cada jurisdicción, donde su financiamiento está previsto en la propuesta de presupuesto (BRASIL, 1990).

La planificación regional integrada establece el CIR como un espacio de articulación, negociación y acuerdo entre las entidades firmantes, permitiendo la identificación, definición de prioridades y negociación de soluciones para la organización de una red regional de acciones y servicios de atención integral y resolutiva de salud de acuerdo con los principios del SUS (BRASIL, 2011).

EI CIR tiene la importancia de consolidar las políticas de salud pública, a través del monitoreo de la Programación Integrada Acordada, a fin de favorecer la construcción del diseño normativo, con definición de flujos y protocolos con miras a la calificación del control social y organización de la gestión compartida de manera ascendente, planificado con definición de recursos financieros y administrativos que contribuyan a la regionalización (BRASIL, 2011).

Los resultados del análisis documental muestran que el CIR es un espacio con potencial para desarrollar capacidad institucional, para la planificación y coordinación territorial regional, orientada a la superación de los intereses corporativos y la creación de una gobernanza local basada en la cogestión y democratización de la decisión y cooperación intergubernamental.

\subsection{ORGANIZACIÓN DE ACCIONES Y SERVICIOS DE ATENCIÓN DE LA SALUD: REGIÓN DE LA SALUD}

El análisis de las resoluciones del CIR muestra que la organización de la atención de salud es una "etapa" constante de discusión en las esferas federativas, evidenciando que las redes de atención de salud son la dirección para el trabajo de acuerdo dentro de las Comisiones Inter-gerenciales (BRASIL, 2011).

RC: 98470

Disponible: https://www.nucleodoconhecimento.com.br/tecnologia-es/comisionintergestora 
La organización de los servicios de salud debe contemplarse en las regiones de salud, que constituyen un espacio geográfico continuo, incorporado por un clúster de municipios, constituido como base territorial para la planificación de la atención de salud, que resulta de la región compartida del financiamiento regional a través de la ejecución de servicios orientados a la gobernanza en la región, a través de la cooperación entre entidades federativas; debe contener acciones de atención primaria, atención psicosocial, vigilancia de la salud, emergencia-emergencia, atención ambulatoria especializada y hospitalaria (BRASIL, 2011).

Para la integración de las acciones y servicios de salud pública en una red regionalizada, es necesario que las entidades federadas trabajen de manera integrada.

Esta agregación de servicios en la región es necesaria para asegurar la integralidad de la atención, a través del flujo de referencia y contrarreferencia, y le corresponde al administrador más amplio responder, en la región, por servicios de mayor complejidad, que requieren escala y otros componentes administrativos y tecnológicos, a los que pueden acceder los municipios de otras entidades federadas (ANDRADE; FRANCISCHETTI, 2019).

Según Santos (2017) la cooperación entre las instancias de acuerdo en el territorio como forma de organizar los espacios de operación del SUS, es un punto clave dado que su singularidad sanitaria, tecnológica y financiera es fundamental en la estructuración del SUS. Sin este formato organizativo, el sistema de salud no era capaz de brindar una atención integral, equitativa y descentralizada.

Como forma de organizar e integrar esta red de servicios dentro del territorio, los firmantes asumen la responsabilidad establecida en el Contrato Organizativo de Acción Pública (COAP), con el propósito de asegurar las acciones y servicios de salud de manera regionalizada y jerárquica, con definición de evaluación ejecutiva, presupuestaria, financiera y de control de evaluación definida entre Estados,

RC: 98470

Disponible: https://www.nucleodoconhecimento.com.br/tecnologia-es/comisionintergestora 
Municipio y Unión, estableciendo metas de salud para el costeo y la inversión que garanticen la atención plena a la población (BRASIL, 1990, 2010, 2011; SANTOS, 2017).

La decisión de calificar la atención y la gestión de la salud involucra aspectos técnicos, éticos y culturales, con la necesidad de cumplir con el pacto político cooperativo entre los órganos de gestión del sistema, expresado por una "fina asociación de técnica y política", para asegurar las inversiones y los recursos necesarios para el cambio (BRASIL, 2010, 2011).

La solución es innovar el proceso de organización del sistema de salud, reordenando acciones y servicios en el desarrollo de Redes Regionalizadas de Atención a la Salud (RAS), para producir un impacto positivo en los indicadores de salud de la población.

El Pacto de Gestión del SUS establece la necesidad de diseñar RAS, organizado en los territorios de las regiones de salud, que sea el espacio privilegiado para la construcción de responsabilidades acordadas y permita la integración de políticas y programas a través de la acción conjunta de los niveles federal, estatal y municipal (BRASIL, 2010).

El Decreto 7508/11, en el Capítulo II, define que el SUS debe estar conformado por una red regionalizada y jerárquica, y que sus regiones de salud deben organizarse para ofrecer servicios de salud articulados en niveles de complejidad creciente, con el propósito de garantizar la atención integral y la atención de salud (BRASIL, 2001).

El Pacto estableció el espacio regional como un lugar privilegiado para la construcción de las responsabilidades acordadas, requiriendo el cumplimiento de las responsabilidades asumidas y las metas acordadas, y cada esfera de gobierno es corresponsabilidad de la gestión del conjunto de políticas, con responsabilidades explícitas (BRASIL, 2006).

RC: 98470

Disponible: https://www.nucleodoconhecimento.com.br/tecnologia-es/comisionintergestora 
Los resultados muestran claramente la organización de los servicios de salud por redes asistenciales y convenios federativos para la ejecución de servicios, contemplando las necesidades de las regiones sanitarias. Según Santos (2017) es necesario actuar de manera integrada y sistémica compartiendo competencias de manera flexible, con el objetivo de asegurar el acuerdo entre las entidades federativas, que influyen en la salud de la población, promoviendo la intersectorialidad, capaces de reducir las desigualdades sociales.

\subsection{FEDERALISMO, COMISIONES INTERGESTIÓN Y FINANCIACIÓN DE LOS SERVICIOS DE SALUD}

El federalismo es una forma de organización del Estado que nace del equilibrio dialéctico entre centralización y descentralización del poder político, correspondiente a la necesidad de mantener la unidad en la diversidad, sin concentrar el poder en un solo núcleo, ni pulverizarlo (DOURADO; ELIAS, 2011).

Según Curvina (2017), el federalismo surgió en 1787, con la Constitución de los Estados Unidos de América. Según el autor, el modelo de federalismo estadounidense fue copiado por los demás países, convergiendo, total o parcialmente, a una dualidad de competencias, caracterizada por la coexistencia de al menos dos esferas constitucionales de poder autónomo, actuando sobre el mismo territorio y la misma población (MENICUCCI, 2019).

En Brasil, el federalismo partía de la existencia de una unidad con poder centralizado, distribuyéndolo entre varias unidades (entidades federadas), sin eliminar el poder central. Este poder delegado a los estados y municipios se establece constitucionalmente a través de la legislación federal, estatal y municipal. Un sistema de federalismo cooperativo, tanto para cuestiones económicas, sociales y culturales, a través de programas y cofinanciación, considerado por Viana et al (2008). "flexibilidad institucional", buscando la cooperación intergubernamental para lograr fines comunes.

RC: 98470

Disponible: https://www.nucleodoconhecimento.com.br/tecnologia-es/comisionintergestora 
Barata (2004) cabe señalar que no existe jerarquía en la organización federal, pues cada esfera de poder corresponde a una competencia determinada, presuponiendo autonomía de las entidades federadas. Aunque el estudio del federalismo pasa por el examen de las especificidades de cada Federación, existen características comunes que representan los fundamentos del Estado Federal.

En el contexto histórico actual, en el que los Estados nacionales tienen el papel preponderante de promover el bienestar social, existe la necesidad de colaboración mutua entre las esferas de gobierno, a fin de lograr objetivos sociales y económicos, resultante de la identificación de que la ejecución de ciertas funciones públicas no puede ser una atribución exclusiva o hegemónica de ninguna de las entidades federadas, división de competencias, cooperación e intereses comunes. Este es el modelo predominante hoy en día, llamado federalismo cooperativo (CURVINA, 2017).

De hecho, la gestión de las políticas públicas es fundamental para el desarrollo de metas y acciones en el ámbito socioeconómico que apunten al bienestar colectivo de la población, a través de la cooperación en las tres esferas de gobierno a través de la construcción de procesos de toma de decisiones y planificados (SOARES, 2018). Estos procesos representan el "dilema inherente entre las negociaciones directas de los gobiernos locales y los incentivos promovidos por los ideats centrales", derivados de los problemas recurrentes representados por la descentralización y la centralización (DOURADO; ELIAS, 2011, p. 206).

Una adhesión permanente de la gestión del SUS es el fortalecimiento de los lazos inter-federativos, necesarios para la consolidación del sistema. La Ley Orgánica de Salud (Ley 8.080 / 90) estableció nuevas estrategias e instrumentos para la consolidación de redes de atención en salud, valorando la construcción de relaciones de colaboración entre entidades federativas a través de COAP y fortaleciendo el rol coordinador de los gestores estatales y de las comisiones intergestoras (BRASIL, 1990).

RC: 98470

Disponible: https://www.nucleodoconhecimento.com.br/tecnologia-es/comisionintergestora 
De hecho, la descentralización busca construir relaciones intergubernamentales en la implementación de políticas públicas y refuerza la importancia del diálogo entre los gobiernos subnacionales y el gobierno nacional en la conducción de la política de salud. Además de producir cambios significativos en el diseño institucional en la organización gubernamental y en el ámbito social con énfasis en la comunicación y el reparto de responsabilidades entre las entidades federativas en la gestión política y financiera de los sistemas locales de salud, ampliando la cobertura y favoreciendo el acceso de la población a los servicios y acciones de salud (MOREIRA; FERRÉ; ANDRADE, 2017).

Es indiscutible que la descentralización va en contra de la asimetría financiera y de gestión de los niveles de gobierno, en la organización y gestión de la red de servicios heterogénea y no institucionalmente integrada con impactos derivados de la aparición de diversos sistemas locales y aislados de servicios de salud, así como en la fragmentación y desorganización de los servicios puestos a disposición de la comunidad (LEVCOVITZ et al, 2001; SANTOS et al, 2015). Otro punto importante causado por la descentralización discutida por los autores está relacionado con el distanciamiento de las agencias gubernamentales responsables del financiamiento de la salud (PINAFO et al, 2020).

La relación entre descentralización, eficiencia y equidad está permeada por complejas redes causales, sin embargo, la evidencia existente indica que, en un país continental como Brasil, que consiste en diversidad espacial, desigualdades territoriales, costos de distribución, intermediación y control superan con creces las ganancias en la gestión de recursos, distribución de bienes y servicios logradas por la administración centralizada (AFFONSO, 1985; LIMA et al, 2015).

De hecho, la concentración geográfica de servicios de mayor complicidad, las diferencias regionales entre poblaciones y las condiciones político-institucionales de las entidades federativas en Brasil, existe una necesidad y uso de los recursos disponibles, con reparto de responsabilidad entre las esferas de gestión, con el fin

RC: 98470

Disponible: https://www.nucleodoconhecimento.com.br/tecnologia-es/comisionintergestora 
de conciliar intereses en conflicto de diferentes regiones, apuntando a la construcción de un modelo cooperativo y financiero en la prestación de servicios de salud (LIMA et al, 2012).

\subsection{COMISIONES DE GOBERNANZA E INTERGESTIÓN REGIONAL - CIR: CANAL DE NEGOCIACIÓN Y DECISIÓN ENTRE LOS GESTORES MUNICIPALES Y EL ESTADO DENTRO DE LA REGIÓN DE LA SALUD}

Los CIR/AL fueron establecidos el 22 de agosto de 2011, y sus reglas los definen como instancias privilegiadas de negociación, articulación, propuesta y acuerdo respecto a los aspectos operativos del SUS dentro de la Región Salud, respetando las definiciones de la Comisión Intermanagers Bipartita (CIB), constituyendo un espacio permanente de cogestión solidaria y cooperativa a nivel regional, a partir de la identificación de una red de servicios de salud organizados, integrados y resolutivos, asegurando la mejora y aplicación de los principios del SUS (COSEMS/AL, 2014).

Integrado por los Secretarios Municipales de Salud y la representación del Secretario de Estado de Salud, que tiene la prerrogativa de coordinar el proceso de acuerdo, sus decisiones se toman por consenso, de acuerdo con lo establecido por el Pacto por la Salud.

En el estado de Alagoas, a través de la aprobación del Plan Maestro de Regionalización (PDR), se redefinió el diseño territorial sanitario, reorganizó en dos Macrorregiones de Salud, subdivididas en diez Regiones Sanitarias, siendo 06 (seis) en la $1^{\text {a }}$ Macrorregión, con sede en Maceió, y 04 (cuatro) en la 2ª Macrorregión, con sede en el municipio de Arapiraca.

Con la misión de gestionar la Política Regional de Salud, a partir del conocimiento de la realidad epidemiológica y socioeconómica del municipio, el territorio y la región, el

$\mathrm{RC}: 98470$

Disponible: https://www.nucleodoconhecimento.com.br/tecnologia-es/comisionintergestora 
CIR coordina la construcción del RAR, asegurando el acceso de la población a los servicios de salud, regulando, monitoreando y evaluando las metas establecidas para los municipios.

Con el objetivo de profundizar el carácter cooperativo de las relaciones intergubernamentales establecidas por el Pacto por la Salud e institucionalizar, en el ámbito de la gestión del SUS, un conjunto de herramientas de gestión y asistencia, el Decreto 7.508 / 2011 define las regiones de salud y las redes de atención de salud. mapa de salud, Listado Nacional de Acciones y Servicios de Salud RENASES, Lista Nacional de Medicamentos Esenciales - RENAME y COAP (BRASIL, 2011). Instituye las instancias de negociación y articulación intergubernamental que actúan en diferentes escalas territoriales (nacional, estatal y regional).

Según la teoría habermasiana, la efectividad de las demandas percibidas en la esfera pública necesita flujos de comunicación y diálogo, es decir, un modelo deliberativo de democracia. En la concepción republicana, el espacio público y político y la sociedad civil como su infraestructura, son puntos estratégicos de organización democrática y comunicativa. Tener la función de asegurar la fuerza integradora y la autonomía democrática de la práctica del entendimiento entre los sujetos (HABERMAS, 1995).

En condiciones estratégicas, los individuos toman decisiones que anticipan las consecuencias de sus acciones, eligen instituciones políticas, en una palabra, actúan políticamente. Y la forma en que actúan juega un papel decisivo en la obtención y mantenimiento de la democracia, para abrir caminos que permitan la comunicación, contribuyendo al bien mayor, al derecho a la salud.

Habermas (1995) desde el punto de vista de la teoría discursiva, la formación democrática de la voluntad se legitima a través de la toma de decisiones y los supuestos comunicativos, que permiten a través de la racionalidad el uso público de

RC: 98470

Disponible: https://www.nucleodoconhecimento.com.br/tecnologia-es/comisionintergestora 
la razón para dialogar con las necesidades de salud de la población con el fin de garantizar procesos justos y cooperativos.

El buen desarrollo cooperativo ocurrido a través del CIR, contribuye a la articulación y regulación de acciones dentro de los territorios a través de la negociación compartida asumida por las entidades federadas en perspectiva solidaria (Lima, 2012; Santos et al, 2014). Por lo tanto, el fortalecimiento de la gobernanza en la región de salud puede reunir lo que la descentralización misma ha fragmentado y supera los desafíos para la integración de la atención organizada en las regiones de salud (LIMA et al, 2016).

Carvalho et al (1995) consideran que, dependiendo de lo establecido en la Constitución Federal, los municipios gozan de autonomía política y administrativa para planificar y administrar sus sistemas locales de salud. Por lo tanto, la única forma de que el Ministerio y las Secretarías de Salud del Estado interfieran en la gestión municipal sería a través de acuerdos y contratos libremente establecidos entre las partes.

En palabras de Noronha et al (2008), el SUS "forma parte de una concepción amplia del derecho a la salud y del rol del Estado en la garantía de este derecho, incorporando en su estructura institucional y de toma de decisiones, espacios e instrumentos para la democratización y el intercambio de la gestión del sistema de salud".

Dadas estas características y el modelo federativo definido constitucionalmente en salud, es especialmente importante el fortalecimiento de la gestión compartida y los instrumentos que pueden favorecerla, entre ellos.

El estudio permitió llegar a varias consideraciones sobre el tema, sin pretender agotarlo, dadas las limitaciones impuestas por la investigación recortada o incluso por el tipo de análisis propuesto por el estudio.

RC: 98470

Disponible: https://www.nucleodoconhecimento.com.br/tecnologia-es/comisionintergestora 


\section{CONCLUSIÓN}

Garantizar el acceso a los servicios de salud, buscando una atención integral requiere de una organización sistémica, proveniente de pactos de cooperación dentro de la gobernanza federativa que apunten a fortalecer la organización de las regiones de salud. Tener al CIR como órgano importante en la coordinación, organización y construcción de los espacios de convenio con miras a la reconstrucción y organización social.

El aprendizaje institucional, resultado de la dinámica de participación en el CIR, fortalece la gestión de los intergerentes y se presenta como un componente importante para la construcción de la red regionalizada.

Esta investigación demuestra que la planificación integral regional, la organización de acciones y servicios, el financiamiento y la gobernanza regional son elementos esenciales en el proceso de regionalización, logrado a través de las deliberaciones del CIR, así como otros puntos acordados en las reuniones, como se describe en las resoluciones, con el enfoque principal en la prestación de servicios de salud disponibles para la población.

La cooperación tiende a favorecer a los municipios que forman parte de una región, porque permite la optimización de recursos, el uso solidario de los medios dispersos entre las entidades federadas, y la garantía de rentabilidad para los municipios con capacidad de asistencia, dejando claro que los CIR/AL son un verdadero espacio local de acuerdo y articulación interfederal.

\section{REFERENCIAS}

AFFONSO, Rui de Brito Álvarez. SILVA, Pedro Luiz Barros. (Org). A federação em perspectiva: ensaios selecionados. São Paulo 10 (3) Edições Fundap; 1995 
[acesso em 3 jul 2020]. Disponível em:https://www.seade.gov.br/wpcontent/uploads/2014/07/v10n3.pdf

ANDRADE, Laurielle de Souza.; FRANCISCHETTI, leda. Referência e contrarreferência compreensões e práticas. Sal. \& Transf. Soc. Florianópolis, v. 10, n. 1/2/3, p. 54-63. 2019 [acesso em 13 jul. 2021]. Disponível em: file://C:/Users/nadja/Dropbox/My\%20PC\%20(LAPTOP-

EA4SVGFV)/Downloads/5281-22616-1-PB.pdf.

BARATA, Luiz Roberto Barradas. TANAKA, Oswaldo Yoshimi. MENDES, José Dínio Vaz. Por um processo de descentralização que consolide os princípios do Sistema Único de Saúde. Epidemiologia e Serviços de Saúde, Brasília, 13 (1) 15 24. 2004 [acesso em 02 jun 2020]. Disponível em: http://scielo.iec.gov.br/pdf/ess/v13n1/v13n1a03.pdf

BRASIL. Conselho Nacional de Secretários de Saúde. Legislação Estruturante do SUS/CONASS. Brasília, 2011. 534. [acesso em 25 jul, 2020]. Disponível em: http://bvsms.saude.gov.br/bvs/publicacoes/para_entender_gestao_sus_v13.pdf

BRASIL. Constituição (1988). Constituição da República Federativa do Brasil. promulgada em 5 de outubro de 1988. Brasília, DF: Senado Federal;1988 [acesso em 10 mai 2020]. Disponível em: https://www2.senado.leg.br/bdsf/bitstream/handle/id/518231/CF88_Livro_EC91_201 6.pdf

BRASIL. Decreto n. 7.508, de 28 de junho de 2011. Regulamenta a Lei n. 8.080, de 19 de setembro de 1990, para dispor sobre a organização do Sistema único de Saúde - SUS, o planejamento da saúde, a assistência à saúde e a articulação interfederativa, e dá outras providencias. Diário Oficial da União, Poder Executivo, Brasília, DF, 29 jun. 2011. Seção 1, p 1. [acesso em 10 fev 2020]. Disponível em http://www.planalto.gov.br/ccivil_03/_Ato2011-2014/2011/Decreto/D7508.htm.

$\mathrm{RC}: 98470$

Disponible: https://www.nucleodoconhecimento.com.br/tecnologia-es/comisionintergestora 
BRASIL. Lei n 8.080, de 19 de setembro de 1990. Lei Orgânica da Saúde. Dispõe sobre as condições para a promoção, proteção e recuperação da saúde, a organização e o funcionamento dos serviços correspondentes e dá outras providências. Brasília, set. 1990. [acesso em 23 abri 2020]. Disponível em: http://www.planalto.gov.br/ccivil_03/leis//8080.htm

BRASIL. Ministério da Saúde. Portaria 4.279, de 30 de dezembro de 2010. Estabelece diretrizes para a organização da Rede de Atenção à Saúde no âmbito do Sistema Único de Saúde (SUS). Diário Oficial da União 31 dez. 2011[acesso em 27 jul 2020]. Disponível em: https://bvsms.saude.gov.br/bvs/saudelegis/gm/2010/prt4279_30_12_2010.html

BRASIL. Ministério da Saúde. Secretaria de Atenção à Saúde. Departamento de Regulação, Avaliação e Controle de Sistemas. Diretrizes para a programação pactuada e integrada da assistência à saúde. Brasília. 2006. [acesso em: 27 jul 2020].

em: http://bvsms.saude.gov.br/bvs/publicacoes/DiretrizesProgPactuadalntegAssistSaude. pdf

BRASIL. Ministério da Saúde. Secretaria de Gestão e Participativa. Contrato Organizativo da Ação Pública da Saúde. Brasília. 2011 [cesso 30 jul 2020]. Disponível em: http://bvsms.saude.gov.br/bvs/publicacoes/contrato_organizativo_acao_publica_sau de.pdf

BRASIL. Resolução no 1 setembro de 2011. Gabinete do Ministério da Saúde. Comissão Intergestores Tripartite. Brasília, [citado 07 Jul. 2020]. Disponível em: https://bvsms.saude.gov.br/bvs/saudelegis/cit/2011/res0001_29_09_2011.html

CARVALHO, Guido Ivan. SANTOS, Lenir. Comentários à lei orgânica da saúde: sistema único de saúde. São Paulo: Ed. Hucitec, 1995 
CONSELHO DE SECRETÁRIOS MUNICIPAIS DE ALAGOAS. 0 papel dos gestores de saúde nas comissões Intergestores regionais. Maceió, 2014 $\begin{array}{llllll}\text { [acesso } & \mathrm{em} & 12 & \mathrm{abr} & 2020] & \text { Disponível }\end{array}$ http://www.cosemsal.org/old/public/documentos/manual-azul-o-papel-dos-gestoresde-saude.pdf

CURVINA, Ana Cristina Carvalho. A regionalização da saúde no federalismo brasileiro. Cad. Ibero-Amer. Dir. Sanit., Brasília, 6(2):42-57, abr./jun, 2017. [acesso em 13 fev 2020]. Disponível em: http://dx.doi.org/10.17566/ciads.v6i2.385

DOURADO, Daniel de Araújo.; ELIAS, Paulo Eduardo Mangeon. Regionalização e dinâmica política do federalismo sanitário brasileiro. Rev. Saúde Pública. São Paulo, v. 45, n. 1, p. 204-11. 2011. [acesso em 05 jul. 2021]. Disponível em: https://www.scielo.br/j/rsp/a/bCWB3sWTXQLXWjjFkcWwgLt/?format=pdf\&lang=pt

DUARTE, Ligia Schiavon.; MENDES, Áquilas Nogueira.; LOUVISONA, Marília, Cristina Prado. O processo de regionalização do SUS e a autonomia municipal no uso dos recursos financeiros: uma análise do estado de São Paulo (2009-2014). Saúde Debate, Rio de Janeiro, v. 42, n. 116, p. 25-37. 2018. [acesso em 10 jul 2020].

https://www.scielo.br/j/sdeb/a/gcPyhjjWdrqZcG9V4nYhPBd/?format=pdf\&lang=pt.

HABERMAS, Jurgen. Três Modelos Normativos de Democracia. Cadernos da Escola do Legislativo, Belo Horizonte, 3 (3)105-122, jan.-jun., 1995.

LEVCOVITZ, Eduardo. LIMA, Luciana Dias de. MACHADO, Cristiane. Vieira. Política de saúde nos anos 90: relações intergovernamentais e o papel das Normas Operacionais Básica. Ciência e Saúde Coletiva, Rio de Janeiro, 6, (2) 269-291, 2001 [acesso em 10 jul 2020]. Disponível em: https://www.scielo.br/pdf/csc/v6n2/7003.pdf. 
LIMA, Luciana Dias de. et al. Regionalização e acesso à saúde nos estados brasileiros: condicionantes históricos e político-institucionais. Ciência e Saúde Coletiva, Rio de Janeiro, 17 (11) 2881-2892, 2012 [acesso 10 jun 2020]. Disponível em: https://www.scielo.br/pdf/csc/v17n11/v17n11a04.pdf

LIMA, Luciana Dias de.; ALBUQUERQUE, Mariana Vercesi.; SCATENA, João Henrique Gurtler. Quem governa e como se governam as regiões e redes de atenção à saúde no Brasil? Contribuições para o estudo da governança regional na saúde. Novos Caminhos, n. 8. 2016. [acesso em 29 fev 2020]. Disponível: https://www.resbr.net.br/wp-content/uploads/2016/02/Novos-Caminhos-8.pdf.

LIMA, Luciana Dias de. et al. Descentralização e regionalização: dinâmica e condicionamento da implantação do pacto pela saúde no Brasil. Ciência \& Saúde Coletiva, n. 17, v. 7, p. 1903-1914, 2012. [acesso em 29 fev. 2020]. Disponível em: https://www.scielo.br/j/csc/a/FyqLwWmbv8WQs8BPW8scpTt/?lang=pt

MENICUCCI, Telma Maria Gonçalves. Regionalização no federalismo brasileiro. Cad. Saúde Pública 2019; 35 Sup 2: e00078419. [acesso em 29 fev 2020]. Disponível: https://www.scielosp.org/article/csp/2019.v35suppl2/e00078419/pt/

MINAYO, Maria Cecília de Souza. 0 desafio do conhecimento. Pesquisa qualitativa em saúde; $12^{\underline{a}}$ ed; São Paulo. Hucitec. 2010. 407p. ISBN 978-85-271-0181-3.

MOREIRA, Laura Monteiro de.; FERRÉ, Felipe.; ANDRADE, Eli lola Gurgel. Financiamento, descentralização e regionalização: transferências federais e as redes de atenção em Minas Gerais, Brasil. Ciência \& Saúde Coletiva, n 22, v. 4, p. 1245-1256, 2017. [acesso em 21 ago 2021]. Disponível em: https://www.scielosp.org/pdf/csc/2017.v22n4/1245-1256/pt.

NORONHA, José Carvalho de. LIMA, Luciana. Dias de. MACHADO, Cristiane Vieira. O Sistema Único de Saúde: SUS. In: Giovanella L. et al. (Org.). Políticas e sistemas de saúde no Brasil. Rio de Janeiro: Fiocruz, 2008. p. 365-394.

RC: 98470

Disponible: https://www.nucleodoconhecimento.com.br/tecnologia-es/comisionintergestora 
PINAFO, Elisangela. et al. Problemas e estratégias de gestão do SUS: a vulnerabilidade dos municípios de pequeno porte. Ciência \& Saúde Coletiva, n2. 25, v. 5, p. 1619-1628, 2020 [acesso em 21 ago 2021]. Disponível em: https://scielosp.org/pdf/csc/2020.v25n5/1619-1628/pt

SANTOS, Adriano Maia dos. GIOVANELLA, Ligia. Governança regional: estratégias e disputas para gestão em saúde. Revista de Saúde Pública, São Paulo, 48 (4) 622-631, 2014[acesso em 10 jun 2020]. Disponível em: https://www.resbr.net.br/wpcontent/uploads/historico/governan\%C3\%A7a_regional.pdf

SANTOS, Lenir. CAMPOS, Gastão Wagner de Souza. SUS Brasil: a região de saúde como caminho saúde e Sociedade, São Paulo, 24 (2) 438-446, 2015 [acesso em 10 jul 2020]. Disponível em: https://www.scielo.br/pdf/sausoc/v24n2/0104-1290-sausoc-24-02-00438.pdf

SANTOS, Lenir. Região de saúde e suas redes de atenção: modelo organizativosistêmico do SUS. Ciência \& Saúde Coletiva, 22(4):1281-1289, 2017. [acesso em 29 jul, 2020]. Disponível em: https://www.scielosp.org/pdf/csc/2017.v22n4/1281$1289 / p t$

SOARES, Marcia Miranda. MACHADO, José Ângelo. Federalismo e políticas públicas. Brasília: Enap, 2018. 112. [acesso em: 29 fev 2020]. ISBN: 978-85-2560081-3Disponível em: https://repositorio.enap.gov.br/bitstream/1/3331/1/Livro_Federalismo\%20e\%20Pol\% C3\%ADticas\%20P\%C3\%BAblicas.pdf.

VIANA, Ana Luiza D’Ávila. LIMA, Luciana Dias. OLIVEIRA, Roberta. Gondim de. Descentralização e federalismo: a política de saúde em novo contexto - lições do caso brasileiro. Ciência e Saúde coletiva. 2002 Jul; 7 (3):493-507. [acesso em 5 mai 2020]. Disponível em: https://www.scielo.br/pdf/csc/v7n3/13027.pdf

RC: 98470

Disponible: https://www.nucleodoconhecimento.com.br/tecnologia-es/comisionintergestora 
VIANA, Ana Luiza D' Ávila. et al. Novas Perspectivas para a regionalização da saúde; São Paulo em perspectiva, 22 (1) 92-106, jan/jun.2008 [acesso em 7 jun 2020].

Disponível em:

http://produtos.seade.gov.br/produtos/spp/v22n01/v22n01_07.pdf

WAGNER, Gastão. Efeitos paradoxais da descentralização do Sistema Único de Saúde do Brasil; In: Fleury S. (Org.). Democracia, descentralização e desenvolvimento: Brasil e Espanha. Rio de Janeiro: FGV, 2006. 417-442. Disponível em https://www.scielo.br/pdf/csc/v17n11/v17n11a04.pdf.

Publicado: Marzo de 2021.

Aprobado: Septiembre de 2021.

RC: 98470

Disponible: https://www.nucleodoconhecimento.com.br/tecnologia-es/comisionintergestora 